\title{
Distant Supervision for Mental Health Management in Social Media: Suicide Risk Classification System Development Study
}

Guanghui $\mathrm{Fu}^{1^{*}}, \mathrm{MSc}$; Changwei Song ${ }^{1 *}$, BSc; Jianqiang $\mathrm{Li}^{1}, \mathrm{PhD}$; Yue $\mathrm{Ma}^{2}, \mathrm{PhD}$; Pan $\mathrm{Chen}^{3}, \mathrm{MSc}$; Ruiqian Wang ${ }^{1}$, BSc; Bing Xiang Yang ${ }^{3}, \mathrm{PhD}$; Zhisheng Huang ${ }^{4}, \mathrm{PhD}$

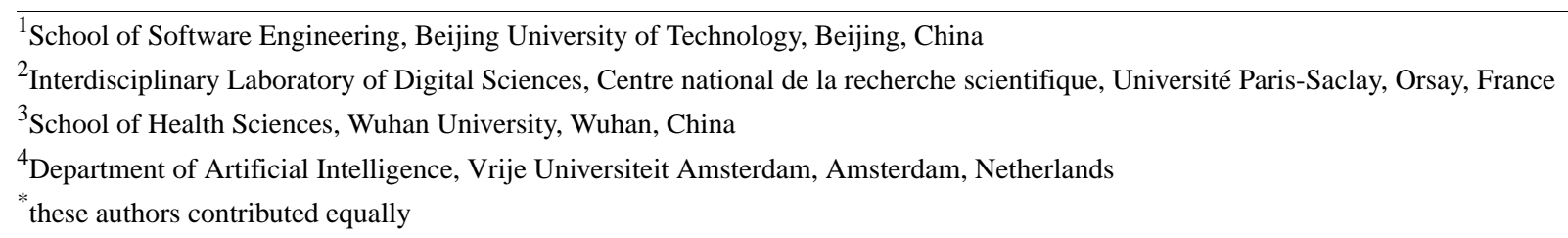

Corresponding Author:

Bing Xiang Yang, $\mathrm{PhD}$

School of Health Sciences

Wuhan University

Wuchang District

Wuhan, 430072

China

Phone: 8615902731922

Email: 00009312@whu.edu.cn

\section{Related Article:}

This is a corrected version. See correction statement in: https://www.jmir.org/2021/9/e33229

\section{Abstract}

Background: Web-based social media provides common people with a platform to express their emotions conveniently and anonymously. There have been nearly 2 million messages in a particular Chinese social media data source, and several thousands more are generated each day. Therefore, it has become impossible to analyze these messages manually. However, these messages have been identified as an important data source for the prevention of suicide related to depression disorder.

Objective: We proposed in this paper a distant supervision approach to developing a system that can automatically identify textual comments that are indicative of a high suicide risk.

Methods: To avoid expensive manual data annotations, we used a knowledge graph method to produce approximate annotations for distant supervision, which provided a basis for a deep learning architecture that was built and refined by interactions with psychology experts. There were three annotation levels, as follows: free annotations (zero cost), easy annotations (by psychology students), and hard annotations (by psychology experts).

Results: Our system was evaluated accordingly and showed that its performance at each level was promising. By combining our system with several important psychology features from user blogs, we obtained a precision of $80.75 \%$, a recall of $75.41 \%$, and an F1 score of $77.98 \%$ for the hardest test data.

Conclusions: In this paper, we proposed a distant supervision approach to develop an automatic system that can classify high and low suicide risk based on social media comments. The model can therefore provide volunteers with early warnings to prevent social media users from committing suicide.

(J Med Internet Res 2021;23(8):e26119) doi: 10.2196/26119

\section{KEYWORDS}

deep learning; distant supervision; mental health; crisis prevention 


\section{Introduction}

\section{Background}

Mental disorders have become a serious problem worldwide, with over 264 million people experiencing depression disorders [1]. A recent large-scale survey in China showed that the lifetime prevalence of depression and anxiety is $6.9 \%$ and $7.6 \%$, respectively [2]. Depression is a leading cause of disability worldwide and contributes greatly to the global burden of diseases [3]. Suicide is the most serious consequence of depression [4]. The latest World Health Organization report showed that close to 800,000 people die by suicide every year. Furthermore, for each suicide, there are more than 20 suicide attempts. Suicides and suicide attempts have a ripple effect that impacts families, friends, colleagues, communities, and societies [5]. Stopping suicides before they are successful is a top priority. With the widespread popularity of the internet and the lack of immediate support in life, people are more inclined to express their emotions - even suicidal thoughts - in web-based communities, such as Weibo (Sina Corporation) and Twitter. Social media users favor these communities due to the anonymity and real-time advantages that they provide [6]. The data from web-based communities are huge in quantity, and it is difficult to analyze these data manually.

Traditional suicide risk assessment studies mainly conduct psychological tests, interviews, and questionnaires, which cost a lot of money. If computer technology can be used to assist suicide risk assessments, we can greatly improve the coverage and efficiency of screening and therefore reduce the number of suicide attempts. In recent years, many deep learning methods have been used for text sentiment analysis. However, these methods require large amounts of labeled data. With regard to the topic of our study, several thousands of comments are generated every day, but only a few (typically, less than 10) are indicative of high suicide risk. It is very time consuming to label so much data, and analyzing low and high suicide risk requires professional knowledge and special training.

In this context, we propose a distant supervision model to reduce the workload of domain experts. We integrated interesting scientific findings from the psychology field into our model. We developed a system that requires no manual annotations and takes into account the feedback of experts to better classify people's suicide risk levels based on the textual comments published on a Chinese social media platform. To avoid expensive manual data annotations, we used a knowledge graph method to produce approximate annotations, which provided a basis for building a deep learning model. The learning model was further refined by interactions with people with different experiences in psychology (beginners and experts) to generate our three data sets-the free annotation (zero cost), easy annotation (labeled by psychology students), and hard annotation (labeled by psychologists) data sets. We built the following three progressive models to fit these data sets: a bidirectional encoder representations from transformers (BERT)-based model, a fine-tuning model, and the psychology+ model. We obtained a precision of $80.75 \%$, a recall of $75.41 \%$, and an $\mathrm{F} 1$ score of $77.98 \%$ for the hard annotation data set, which was the hardest data set among the three data sets. This system reports on suicide risk assessments to volunteers from the Tree Hole Rescue Team [7] for web-based crisis prevention in China.

We first introduce the background of our research and discuss related work in the Introduction section. In the Methods section, we describe the task and introduce our workflow. In the Results section, we describe our data sets and a series of experimental results to prove the performance of our method. In the Discussion section, we summarize a series of open problems in this study and put forward some thoughts on future research directions. We summarize the whole paper in the final Conclusion section.

\section{Related Work}

\section{Distant Supervision}

Distant supervision is a method for using prior knowledge to generate noise labels (data containing wrong labels), which can help with avoiding a lot of manual labeling. In 2009, Mintz et al [8] first proposed the distant supervision method and used it for relation extraction. They generated a large amount of noise labels by mapping an existing knowledge base to rich unstructured text. Thus, they were able to train a good relationship extractor. Some sentiment classification methods also involve the distant supervision method. Go et al [9] proposed a method for automatically classifying sentiment in Twitter messages. They first used the emoticons in the text to generate labels and then used machine learning algorithms for classification. Purver and Battersby [10] and Suttles and Ide [11] used emoticons and hashtags or emojis in Twitter data to generate noise labels. Sahni et al [12] used half of a data set for comparisons with a baseline model and achieved a $2 \%$ to $3 \%$ higher accuracy than the baseline model. Camacho-Collados et al [13] proposed a simple, distant, supervision-based postprocessing step for using noisy, user-generated text to learn cross-lingual embeddings. They used English data to train classifiers for other languages. Purver and Battersby [10] used tag symbols and topic tags as weak tag training models. Without human intervention, the models were used to detect multiple types of emotions in Twitter messages. Mohammed et al [14] proposed a novel distant supervision technique for automatically gathering noisy topic category annotations from topically focused streams.

Most related research is based on the same annotation standard and eliminates the differences between different data through distance supervision. In our study, different levels of annotators (from nonprofessional to professional) provided different standards. Our method can be used with different levels of annotators (from basic algorithms to domain experts) to train models and obtain performance improvements to deal with real scenarios.

\section{Deep Learning-Based Text Sentiment Analysis}

Text sentiment analysis is the task of detecting sentiment information contained in text through a computer program. A basic task in sentiment analysis is classifying the polarity of a given text, that is, whether the expressed opinion in the text is positive, negative, or neutral. In advanced cases, polarity can refer to emotional states such as anger, sadness, and happiness. 
Sentiment analyses have been applied in marketing, customer service, and clinical medicine. Different from classical text sentiment analysis, our task of classifying high and low suicide risk on the basis of a given text was mainly based on whether users had decided a specific suicide method and a determinate suicide plan. Negative polarity and the emotional states of sadness and anger unnecessarily imply a high risk.

In recent years, many deep learning methods have been used for text sentiment analysis. Kim [15] used the word2vec method to convert sentences into sentence vectors and input the vectors into a convolutional neural network for sentiment analysis and question classification. Kalchbrenr et al [16] designed a dynamic convolutional neural network for binary and multi-class sentiment prediction. The model can handle input sentences of different lengths and can explicitly capture short and long relationships. Dieng et al [17] proposed a recurrent neural network for the sentiment analysis of movie reviews, which could capture the long-term dependency of text sentiment analysis. However, these methods all use word embedding for word vector representation, which cannot solve the problem of polysemy in reality. In 2018, Devlin et al [18] proposed a BERT-based, pretrained language model for dynamically obtaining encoded word vectors according to context. The BERT-based, pretrained language model uses a bidirectional transformer [19] as a feature extractor. Compared to traditional word embedding methods, such as Word2Vec [20-22] and Glove [23], the BERT method is better for capturing the representation of word and sentence levels. The BERT model is one of the most state-of-the-art pretraining models. Our final model was modified based on BERT, integrated the psychology features (Textbox 1) of users, and provided a more comprehensive suicide risk detection system.

Textbox 1. Psychology features and their definitions.

\section{Self-description length}

- The number of bytes that a user's homepage simply introduces to them

\section{Weibo originality rate}

- The ratio of the amount of original Weibo data to the total amount of Weibo data

\section{Weibo link rate}

- The ratio of the number of linked Weibo pages to the total number of Weibo pages

\section{Weibo interaction rate}

- The average number of mentions of other people in a user's Weibo account

\section{Collective attention rate}

- The average number of times each Weibo user uses first-person plural terms

\section{Self-focus rate}

- The average number of times each Weibo user uses first-person singular terms

\section{Nighttime activity rate}

- The ratio of the number of active users on Weibo from 10 PM to 6 AM to the total number of Weibo users

\section{Social activity rate}

- The ratio of the number of mutual attentions and the number of followers that a Weibo user has to those of other users

\section{Willingness to express rate}

- The ratio of the number of a user's Weibo comments to the number of their likes

\section{Social support rate}

- The ratio of the number of a user's Weibo comments to the total number of Weibo comments

\section{Methods}

\section{Study Design}

As previously mentioned, the information for classifying high and low suicide risk in our task is different from those used in the classical sentiment analysis tasks. In this study, to avoid the high cost of creating manually annotated data, we used a distant supervision approach that does not require manual annotations. Domain experts can use this approach to provide a small amount of annotations, which provides a basis for further improving a model by taking into account the experts' feedback. The flowchart of our method is shown in Figure 1. 
The method was divided into two parts - automated annotation generation (via the knowledge graph rules in Figure 1) and deep learning-based classification (the three models in Figure 1).

For automated annotation generation, a set of semantic rules was constructed based on an ontology for the field of crisis prevention to generate the free annotation data set. These automated (possibly erroneous) annotations were then used to supervise the deep learning models.

We then build a BERT-based model based on the free annotation data set. This model was used to classify new texts that were further corrected by psychology students to generate the easy annotation data set. This data set had comparable amounts of high and low crisis risk data. It should be noted that without the assistance of a computer algorithm, it would have been a massive challenge for humans to provide such a balanced data set because the percentage of high-risk messages was quite low, as mentioned above. We used the easy annotation data set to fine-tune this basic learning model and develop the fine-tuning model, which took into account the knowledge in the easy annotations. In parallel, a psychology expert assisted with providing the hard annotation data set, which is of much smaller size than the easy annotation data set due to its cost. Finally, we improved our model by using the hard annotation data set and extra psychology features to obtain the final psychology+ model.

Our models continually fitted 3 data sets. These three data sets were labeled by psychology practitioners of different levels. As the standards for the labeling process gradually became stricter, the models became more accurate. The final model (psychology+ model) combined the prior knowledge obtained from the precedent models and fused prior domain knowledge. Improved performance was achieved under the premise of using a small amount of manually annotated data.

Figure 1. Flowchart of our method. As the labeling process became stricter, we continued to improve our model's performance. BERT: bidirectional encoder representations from transformers.

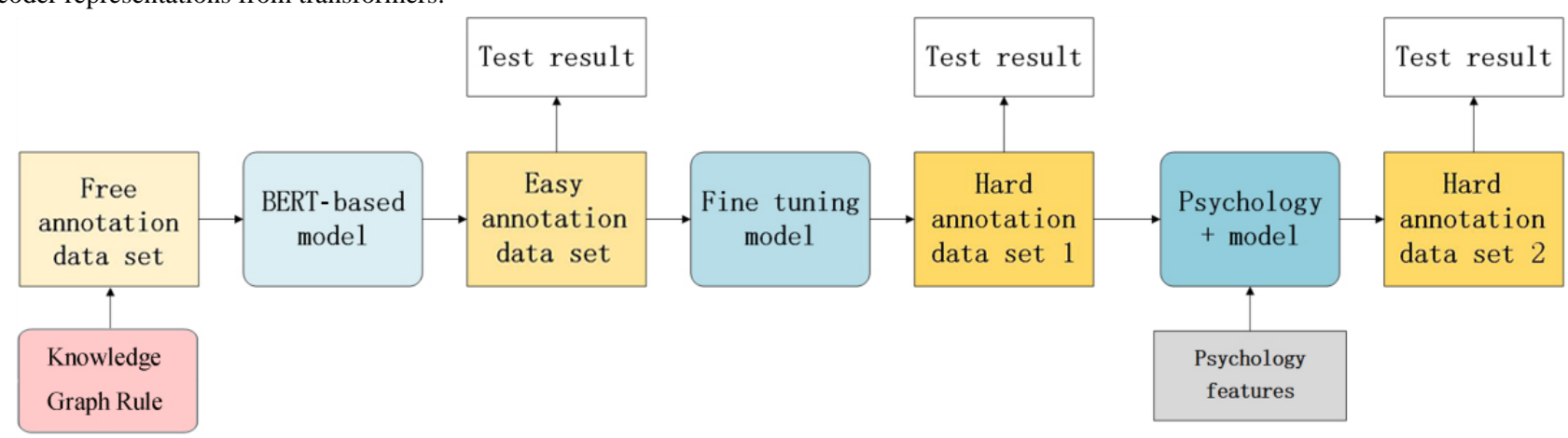

\section{Task Definition}

Given a textual sentence $(s)$ published on a web-based media platform by a user $(u)$, our task was to predict if the user $(u)$ has a high suicide risk. If this is the case, a crisis intervention will be provided to save the user's life. This is a challenging task, and even a person with professional knowledge has to be careful to avoid mistakes. Furthermore, the huge amount of comments produced daily makes manual analysis impossible and costly.
With regard to the definitions of high and low risk, Huang et al [24] proposed a grading standard based on the certainty of planned suicide methods and the urgency of the action, thereby obtaining 10 risk levels, as shown in Textbox 2. We defined level 6 and above as "high risk" and the rest as "low risk." Furthermore, social media may contain a lot of irrelevant comments. Therefore, we created level 0 and defined it as "no risk." 
Textbox 2. Suicide risk levels and judgement criteria.

Level 10

- $\quad$ Suicide may be ongoing

Level 9

- $\quad$ Suicide method has been determined

- Person may commit suicide in the near future

Level 8

- Suicide has been planned

- Date of suicide has been roughly determined

Level 7

- Suicide method has been determined but not the suicide date

Level 6

- $\quad$ Suicide method is planned

- $\quad$ Suicide method is unknown

Level 5

- Strong suicide desire

- Unclear mode of suicide

Level 4

- $\quad$ Suicide wish has been expressed

- Suicide method and plan are unclear

Level 3

- Strong survival pain

- No expression of suicidal wish

Level 2

- The pain of survival has been clearly expressed

- No suicidal desire

Level 1

- Expression of survival pain

- Expression of suicide desire

Level 0

- No expression of survival pain

\section{Knowledge Graph and Reasoning Rules}

We created a knowledge graph that was used to construct a free annotation data set. The Tree Hole knowledge graph contains four independent ontologies - the suicide ontology, time ontology, space ontology, and wish ontology. The suicide ontology consists of two major categories - suicide methods (eg, cutting the wrist and burning charcoal) and suicide plans (eg, buying drugs and meeting with suicide partners). The time ontology covers absolute time concepts, such as calendar days and holidays, and relative time concepts, such as the present, future, and past. The space ontology describes related concepts of spatial geography. The wish ontology was mainly used to analyze the subjective suicidal wishes of a specific group of people and to exclude them from people without subjective suicidal wishes. An excerpt of the Tree Hole knowledge graph can be found in Figure 2. 
Figure 2. An excerpt of the Tree Hole knowledge graph.

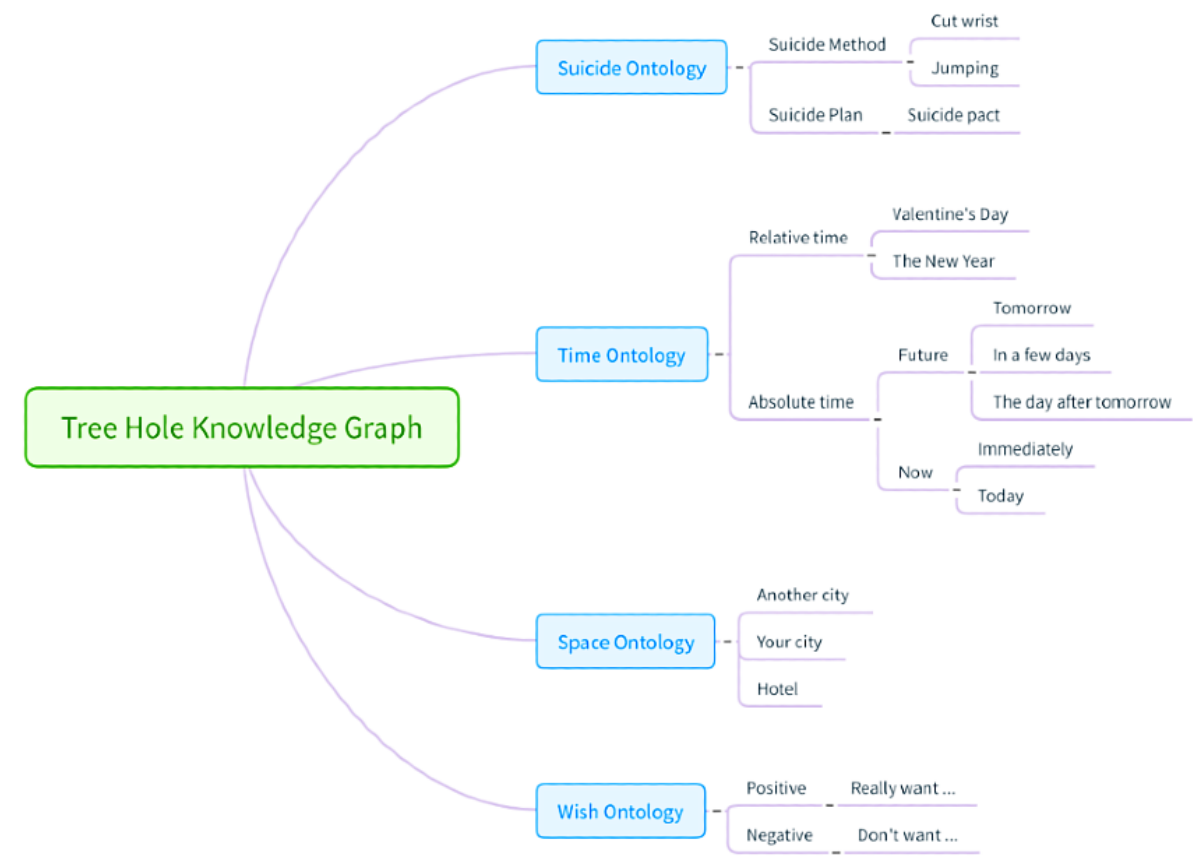

Once the knowledge graph was created, we constructed prolog rules based on the definite clause grammar (DCG) and DCG transformation rules. To take into account the domain knowledge for reasoning and determining the risk level, the DCG rules integrate the relevant conceptual information from the Tree Hole knowledge graph. For example, the definition for suicide risk level 8 is that the suicide plan has been determined and the suicide date has been roughly determined. This can be formally described by the logic program rules in Textbox 3, which use two predicates-rdfsSubClass Of and uninterestedText. The predicate $r d f$ SSubClass $O f$ was used to decide if a textual fragment mentions some sort of suicide method (or time) concept of suicideOntology (or timeOntology) in the Tree Hole knowledge graph, and uninterestedText was used to verify that such a mention was not negated by a negative expression in the text. More details can be found in other studies [24,25].

Textbox 3. Logic program rules for suicide risk level 8.

\section{Program rules}

- $\quad$ statement(suicideRisk(8,[Plan, Time])) $\rightarrow \quad$ uninterestedText(_L1), rdfsSubclassOf(Time,future,timeOntology), uninterestedText(_L2), rdfsSubclassOf(Plan, suicidePlan, suicideOntology), uninterestedText(_L3)

- $\quad$ statement(suicideRisk(8, [Plan, Time])) $\rightarrow$ uninterestedText(_L1), rdfsSubclassOf(Plan, suicidePlan, suicideOntology), uninterestedText(_L2), rdfsSubclassOf(Time, future, timeOntology), uninterestedText(_L3).

\section{BERT-Based Model}

We used the data set generated by Tree Hole knowledge graph method to build the BERT-based model. We used BERT to obtain sentence vectors from free annotation data. The size of each sentence vector was 768 dimensions. We added a dropout function to this sentence vector to avoid overfitting. Afterward, we added a fully connected layer to classify comments that were indicative of high and low suicide probabilities. We used the sigmoid function as the activation function of the output layer. The parameters of the BERT layer and the fully connected layer participated in the training at the same time. The architecture of model 1 is shown in Figure 3. 
Figure 3. Architecture of our BERT-based model. BERT: bidirectional encoder representations from transformers .

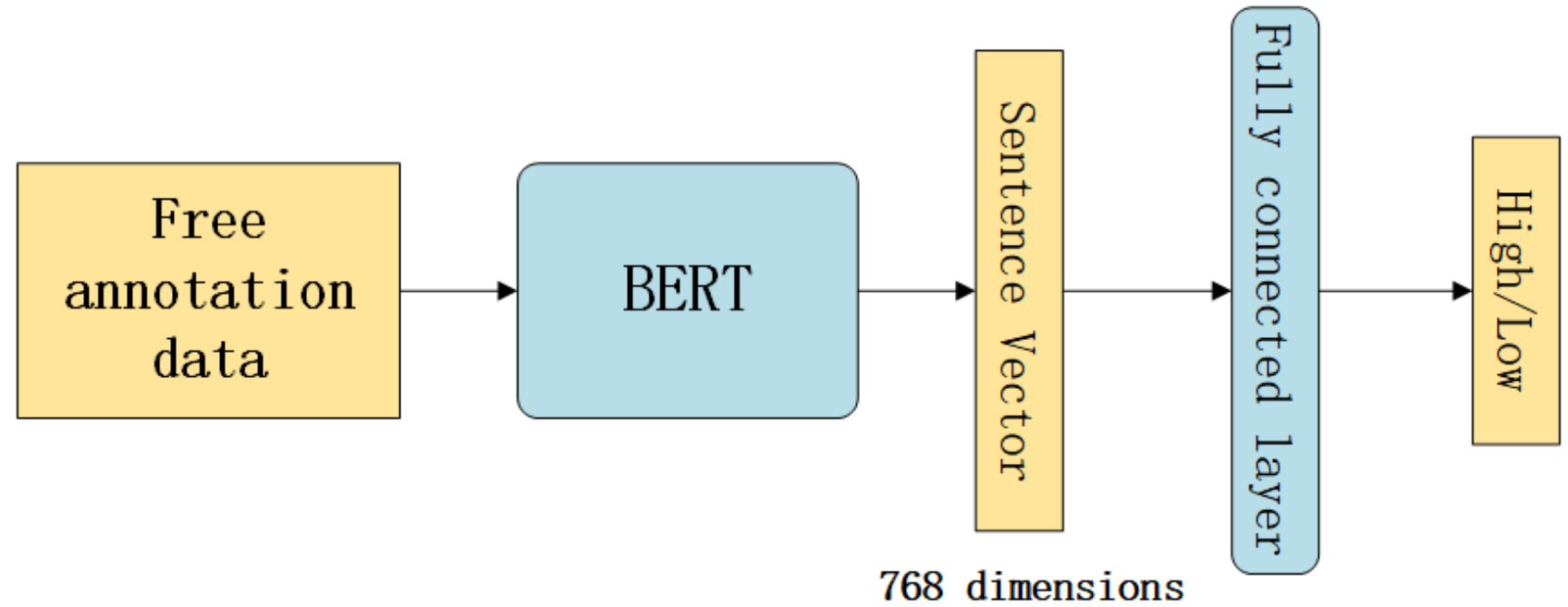

\section{Fine-Tuning Model}

We used BERT-trained (the first model) to obtain 768D sentence vectors from the easy annotation data set. We added 3 fully connected layers to the sentence vectors. The input and output of the first 2 fully connected layers had 768 dimensions, and we used the ReLU function [26] as their activation function.
We also added a dropout function and batch normalization function [27] after each activation function to prevent overfitting. We used the sigmoid function as the activation function of the output layer. Different from the first model, the fine-tuning model did not train the BERT layer but only trained the previous fully connected layer. The structure of model 2 is shown in Figure 4.

Figure 4. Architecture of our fine-tuning model. BERT: bidirectional encoder representations from transformers.

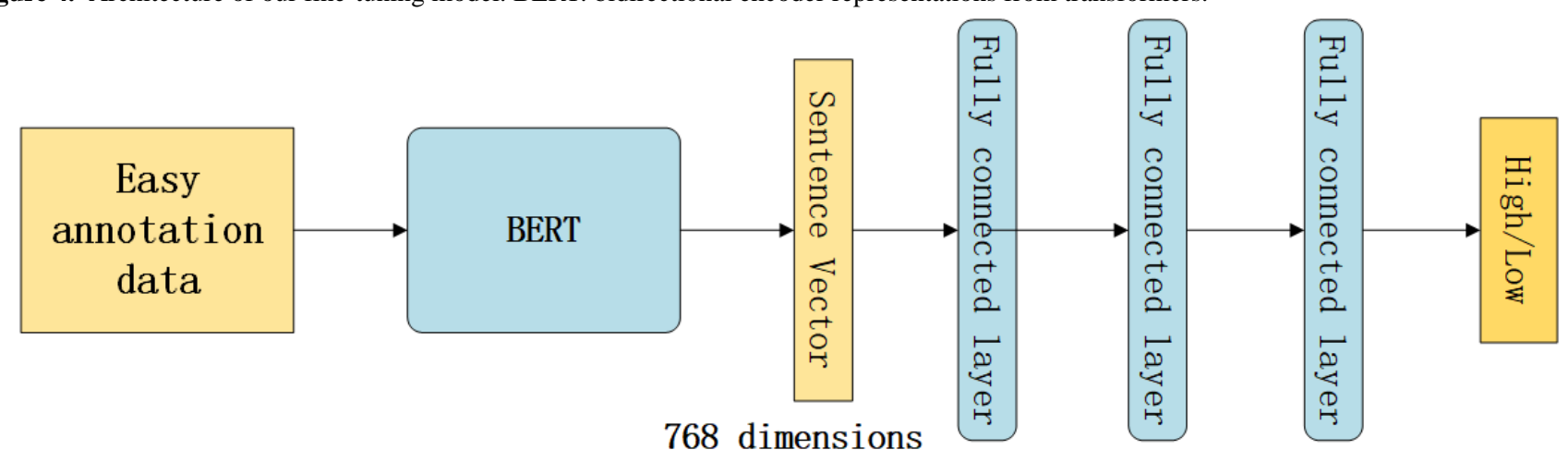

\section{Psychology + Model}

This model combined deep learning and psychology features. According to a psychology study, people with a high suicide risk have a higher degree of self-concern than those with a low suicide risk, that is, they may be more focused on themselves rather than their surroundings [28]. Social network behaviors can reflect individuals' personality traits [29]. Gosling et al [30] found that people with extraverted personality traits use social networks more frequently, have higher engagement, and are more active compared to introverts. To take advantage of these scientific findings, we built the psychology+ model, as described below.

We analyzed the 10 psychology features defined in Textbox 1. According to psychological research, people with a high and low suicide risk have significantly different behaviors with regard to these 10D features [28-32]. We further analyzed and obtained Weibo users' psychology features and integrated them into our final model. These 10 features were represented as a $10 \mathrm{D}$ vector. Afterward, we used the BERT-trained model (the first model) to obtain $768 \mathrm{D}$ sentence vectors from hard annotation data. We used 2 fully connected layers to reduce the sentence vector to 64 dimensions. Our choice of using a 64D vector was based on experiments. We experimented with 256 , $128,64,32$ dimensions, and the best results were obtained with 64 dimensions. We combined the 64D sentence vector and 10D psychological feature vector to form a 74D vector. Finally, we used the fully connected layer as a classifier and the sigmoid function as the activation function to classify high and low suicide risk. This model structure is shown in Figure 5. 
Figure 5. Architecture of our psychology+ model. BERT: bidirectional encoder representations from transformers.

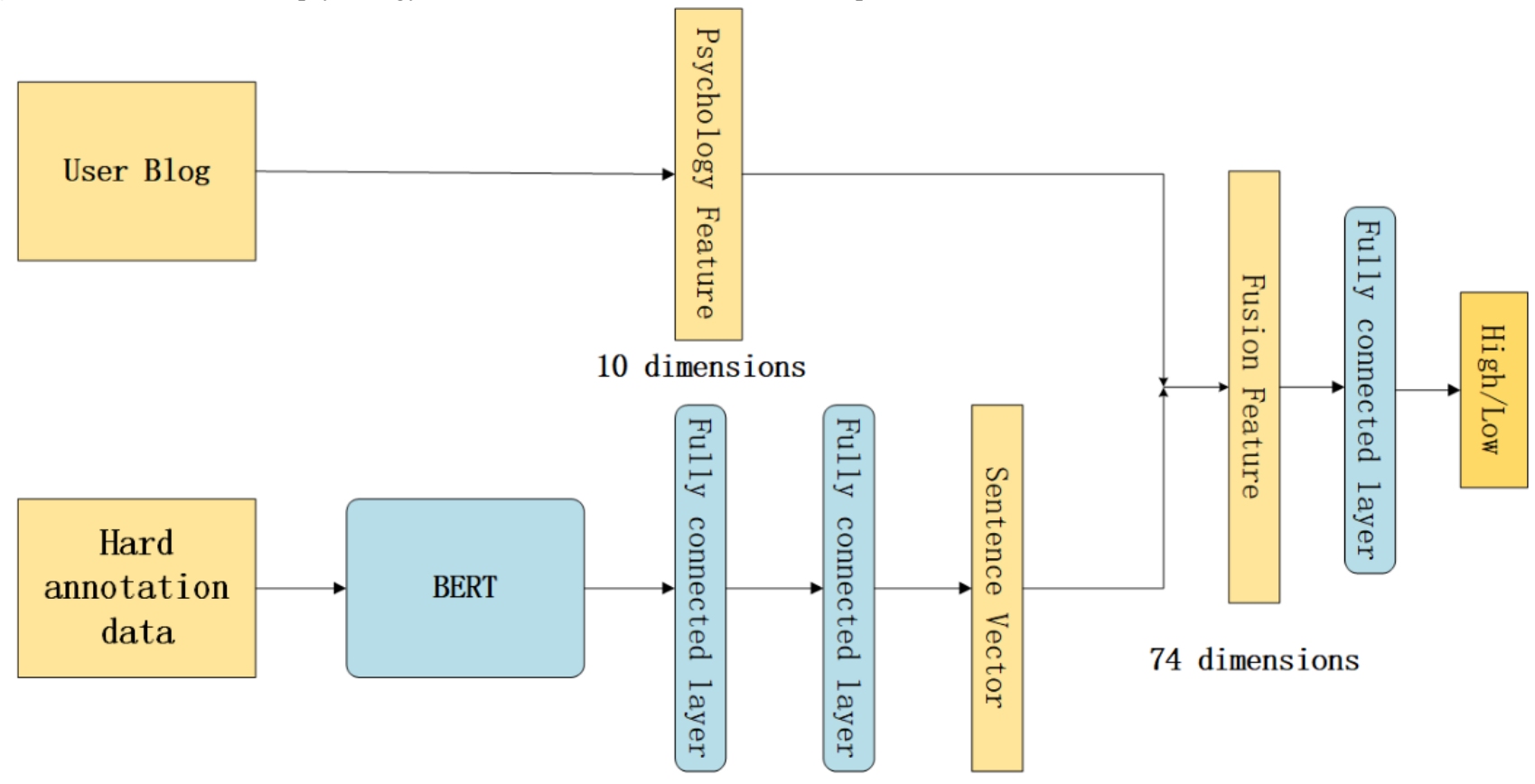

64 dimensions

\section{Results}

\section{Data Set}

In this study, data were obtained from the comments of the Zoufan Weibo account [33]. In addition, we performed the following preprocessing methods for the data sets: the removal of duplicate data, the removal of meaningless emojis, and the removal of too short sentences ( $\leq 5$ words). As previously explained, we constructed the following four data sets (our annotation data sets can be made available upon request for research purposes):
- the free annotation data set, which was generated by using knowledge graph method

- the easy annotation data set, which was labeled by a psychology student

- $\quad$ hard annotation data sets 1 and 2, which were labeled by a psychologist

These data sets were used to train and test our models. The data distribution can be seen in Table 1.

For the psychology+ model, the psychology features were extracted from each user's Weibo page [34].

Table 1. Comment distributions in each data set.

\begin{tabular}{lll}
\hline Data set & Comments indicating a high suicide risk, $\mathrm{n}$ & Comments indicating a low suicide risk, $\mathrm{n}$ \\
\hline Free annotation data set & 3630 & 3220 \\
Easy annotation data set & 6659 & 8657 \\
Hard annotation data set 1 & 813 & 645 \\
Hard annotation data set 2 & 599 & 601 \\
\hline
\end{tabular}

\section{Evaluation}

We evaluated the three learning models that were built based on the automatically generated annotation data set. We found that the free and easy annotation data sets resulted in a simple classification task that could be solved well by the BERT-based model and the fine-tuning model, respectively. However, the hard annotation data set resulted in a much harder task for which our psychology+ model could achieve a promising performance.

\section{Experimental Setup}

For the basic BERT-based model, we used the pretrained Chinese model released by Google. The model uses a 12-layer transformer with about 110 million parameters. The optimizer uses the Adam method [35], and the learning rate was set to 0.00002 . The maximum length of inputted characters is 128 . The batch sizes of the training set, validation set, and test set were 8,16 , and 16 , respectively. At the fully connected layer, the dropout was set to 0.1 . We built our model by using a GTX 2080Ti graphics processing unit, and the deep learning framework we used was PyTorch [36]. In the evaluation experiments, we used F1 scores, precision, recall, and the accuracy of high-risk classifications as the evaluation metrics. The F1 score is the harmonic mean of precision and recall, and it was the most important evaluation metric in our study. 


\section{Evaluation of the BERT-Based Model}

We performed fivefold cross-validation for 6850 comments (3630 comments indicating a high suicide risk and 3220 indicating a low suicide risk; Table 1) and thus obtained 5480 comments for training and 1370 comments for testing. We report in Table 2 the evaluation results of the model that was based on the free annotation data set and the easy annotation data set.
We found that the values of all evaluations metrics for the free annotation data set were higher than 0.98 . This shows that this simple model can simulate the knowledge graph-based approach well. The model's performance based on the easy annotation data set was lower, particularly in terms of precision (0.9899 vs 0.8367 ), indicating that the model needs further improvement to analyze behavior just as well as psychology students for the annotation task.

Table 2. Bidirectional encoder representations from transformers-based model.

\begin{tabular}{lllll}
\hline Data set & F1 score & Precision & \multicolumn{2}{c}{ Recall } \\
\hline Free annotation data set & 0.9864 & 0.9899 & 0.9829 \\
Easy annotation data set & 0.9111 & 0.8367 & 0.9998 \\
\hline
\end{tabular}

\section{Evaluation of the Fine-Tuning Model}

According to the fivefold cross-validation, we separated 15,316 comments (6659 comments indicating a high suicide risk and 8657 indicating a low suicide risk; Table 1) into the training set or the test set, which contained 12,252 and 3064 comments, respectively. We also evaluated the performance of the model based on hard annotation data sets 1 and 2 .

The results can be seen in Table 3. Clearly, by fine-tuning the model, the performance of the model improved (compared to the BERT-based model) in terms of F1 score (0.9111 vs 0.9214), precision (0.8367 vs 0.9241), and accuracy (0.9151 vs 0.9218). However, the recall value dropped a little bit (0.9998 vs 0.9282$)$.
In contrast to the performance of the model based on the easy annotation data set, the results for the 2 hard annotation data sets were unsatisfactory $(<0.6$ in most cases). This meant that the model needed further improvement. Intuitively, we could have performed a similar fine-tuning process with the hard annotations. However, as seen in Table 1, the hard annotation data sets were much smaller than the easy annotation data set. This was due to the high cost of the hard annotations. Therefore, we created the psychology+ model by taking into account certain psychological knowledge in order to avoid the requirement of large amounts of annotated data.

Table 3. Fine-tuning model.

\begin{tabular}{lllll}
\hline Data set & F1 score & Precision & Recall & Accuracy \\
\hline Easy annotation data set & 0.9214 & 0.9241 & 0.9282 & 0.9218 \\
Hard annotation data set 1 & 0.7281 & 0.5815 & 0.9734 & 0.5942 \\
Hard annotation data set 2 & 0.6753 & 0.5131 & 0.9877 & 0.5239 \\
\hline
\end{tabular}

\section{Evaluation of the Psychology+ Model}

According to fivefold cross-validation, we separated 1458 comments (813 comments indicating a high suicide risk and 645 indicating a low suicide risk from hard annotation data set 1; Table 1) into the training set or the validation set, which contained 1167 and 291 comments, respectively. Hard annotation data set 2 was reserved mainly for testing. We report the evaluation results for these two hard annotation data sets in Table 4. We obtained an accuracy of $78.68 \%$ for hard annotation data set 2. Compared to the fine-tuning model, the F1 score of the psychology+ model based on hard annotation data sets 1 and 2 improved by $7.88 \%$ and $10.45 \%$, respectively.

Table 4. Psychology+ model.

\begin{tabular}{lllll}
\hline Data set & F1 score & Precision & Recall & Accuracy \\
\hline Hard annotation data set 1 & 0.8069 & 0.8067 & 0.8072 & 0.8105 \\
Hard annotation data set 2 & 0.7798 & 0.8075 & 0.7541 & 0.7868 \\
\hline
\end{tabular}

\section{Discussion}

\section{Principal Findings}

\section{Summary of Findings}

In this study, we examined three types of annotation data sets - the free annotation, easy annotation, and hard annotation data sets. As seen in Table 2 and Table 3, free annotations and easy annotations can be generated by deep learning models of high quality. Even though we achieved promising results from the hard annotation data sets, the quality of the results is still imperfect. Indeed, our task was exposed to some challenges due to several characteristics of the data.

We consider the F1 score to be the most important evaluation metric. In follow-up work, we will invite more volunteers to manually evaluate our model based on actual situations. 


\section{Obscure Expression}

With regard to the two sentences in Figure 6, without considering the context of the task, neither of these two statements are indicative of a high risk of suicide. However, due to the nature of our data source, both sentences were classified as high risk by psychological experts. The first sentence expresses the result of someone cutting their wrist, but in terms of our context, the sentence was used to comment on a suicide attempt (cutting wrist), indicating that the attempt has been taken. Therefore, a prevention intervention should be provided. The second sentence is about making an appointment with other people to die together. In the context of our data source, it can be considered that the date of the suicide was about to be determined, resulting in a high risk level of between 7 and 8 .

Figure 6. Examples of comments that express a high suicide risk in an obscure way.

\title{
别割了, 很疼的, 又死不了 \\ Don't cut yourself. It hurts and you won't die.
}

\section{去哪个城市一起? \\ Which city shall we go together?}

\section{Ultrashort Text}

Short texts can also indicate a high suicide risk. For example, for the three short sentences in Figure 7, the first two are indicative of a low suicide risk, but the third is indicative of a high suicide risk. The is because the first two expressions indicate that the plan is still under consideration. However, the third indicates that the plan has been restricted to two possible suicide methods. Such ultrashort texts are difficult to classify, and successful classification needs the combination of more specific context and domain knowledge.

Figure 7. Ultrashort texts that are indicative of different suicide risk levels.

\section{跳楼? \\ Jump off the building? \\ 烧炭? \\ Burn charcoal? \\ 跳河还是跳楼呢? \\ Jumping into the river or jumping off the building?}

\section{Long Text With Contradictions}

contradictory, redundant, and possibly incomprehensible

Some people express their feelings by telling their own stories, expressions and sentences also make classification difficult. as illustrated by the examples given in Figure 8. These long,

Figure 8. Examples of long texts for which the suicide intention level is hard to capture.

\begin{abstract}
天天在耳边听着想死两个字。我真的也㥿不住了。明知道一个是无病呻吟, 一个是真的想死...可是 就是忍不住的烦, 忍不住就想离开, 想死。但是现实却又一切是那么美满, 让我不能死, 不敢死。 怕让那些看重我的人伤心难过, 我不能自私白发人送黑发人, 但是又真的謷不住觉得自己活着就是 个过错没活着的意义.

Every day I heard the words "going to die" in my head. I really can't take it anymore. I know that on the one side, I am moaning about imaginary illness, and on the other hand, I really want to die... I can't help being bored and I am willing to leave the world. But the reality is also so wonderful, the fact that makes me barely dare to die. I'm afraid to make my beloved ones sad. I can't be so selfish to let my parents watch my death. But I can't help feeling that it's meaningless for me to be alive.
\end{abstract}

\section{最近在绝食, 整天整天地让自己躺在床上不动已经快半个月了, 不想跟我妈妈说话也不想跟我妹妹 说话, 所以我也不想吃饭, 但是我仍旧守着洗碗的承诺, 每天晚上9点过去洗碗, 今天是绝食的第 三天, 我腿好疼, 快要站不起来了, 第二天洗碗的时候感觉我的腿一直在打颤, 我好难过又好愤怒 I have been on a hunger strike recently. I have been lying in bed all day long for almost half a month. I don't want to talk to my mother or my sister. I don't want to eat, but I still keep my promise to wash dishes. I go to wash dishes every night at $9 \mathrm{pm}$. Today is the third day of my hunger strike. My leg hurts and I can't stand up anymore. When I was washing the dishes on the second day, I felt my legs trembling all the time. I am so sad and angry.}

\section{Conclusion}

In this paper, we proposed a distant supervision approach to develop an automatic system that can classify high and low suicide risk based on social media comments. We constructed 3 data sets of different levels (free, easy, and hard) via interactions with psychologists who were assisted by our models. 
Although a deep learning model has excellent performance in different domains, it requires a lot of annotations to train a reliable model. Per our study, ordinary people cannot accurately label data. Only people who have been professional trained can accurately classify the suicide risk expressed in comments in accordance with standard methods. This makes it difficult to obtain large-scale annotations. Per our processing steps, we first used a basic algorithm to generate annotations for training the baseline model. Afterward, we invited people with different psychology knowledge levels to provide a small number of annotations. Then, based on domain knowledge, we extracted users' multidimensional psychological features and integrated them into our final model (the psychology+ model). Our operating steps greatly improved the efficiency of our model.
Only 1458 professional labels were required to train a model that could analyze real situations. It would have been impossible to train a reliable model with just over 1000 data points if we did not conduct the previous steps.

In future work, we will combine actual work experiences and cooperate with psychologists to propose more suitable suicide classification standards and provide immediate warnings for upcoming emergencies. Although our model could meet actual standard requirements, its run time was relatively slow (274 comments/minute) due to the large number of model parameters. Further, although the model's efficiency could meet people's daily needs, we still hope to develop a more lightweight model for dealing with certain data produced in special situations, such as short-term, large-scale comments.

\section{Acknowledgments}

This study is supported by the National Natural Science Foundation of China (No. 72174152) and the Project of Humanities and Social Sciences of the Ministry of Education in China (The Proactive Levelled Intervention for Social Network Users' Emotional Crisis-an Automatic Crisis Balance Analysis Model, 20YJCZH204).

\section{Conflicts of Interest}

None declared.

\section{References}

1. Depression. World Health Organization. 2020 Jan 30. URL: https://www.who.int/news-room/fact-sheets/detail/depression [accessed 2020-01-30]

2. Huang Y, Wang Y, Wang H, Liu Z, Yu X, Yan J, et al. Prevalence of mental disorders in China: a cross-sectional epidemiological study. Lancet Psychiatry 2019 Mar;6(3):211-224. [doi: 10.1016/S2215-0366(18)30511-X] [Medline: 30792114

3. World Health Organization. Mental Health Action Plan 2013 - 2020. Geneva, Switzerland: World Health Organization; 2013.

4. Li H, Luo X, Ke X, Dai Q, Zheng W, Zhang C, et al. Major depressive disorder and suicide risk among adult outpatients at several general hospitals in a Chinese Han population. PLoS One 2017 Oct 10;12(10):e0186143. [doi: 10.1371/journal.pone.0186143] [Medline: 29016669]

5. Suicide. World Health Organization. URL: https://www.who.int/news-room/fact-sheets/detail/suicide [accessed 2019-09-02]

6. Chen P, Qian Y, Huang Z, Zhao C, Liu Z, Yang B, et al. Negative emotional characteristics of weibo "tree hole" users. Zhongguo Xinliweisheng Zazhi 2020;5:437-444.

7. Tree Hole Rescue Team. Weibo. URL: https://weibo.com/u/5133305819 [accessed 2021-07-06]

8. Mintz M, Bills S, Snow R, Jurafsky D. Distant supervision for relation extraction without labeled data. 2009 Presented at: Joint Conference of the 47th Annual Meeting of the ACL and the 4th International Joint Conference on Natural Language Processing of the AFNLP; August 2-7, 2009; Suntec, Singapore p. 1003-1011. [doi: 10.3115/1690219.1690287]

9. Go A, Bhayani R, Huang L. Twitter sentiment classification using distant supervision. Stanford University. URL: https:/ /www-cs.stanford.edu/people/alecmgo/papers/TwitterDistantSupervision09.pdf [accessed 2021-06-29]

10. Purver M, Battersby S. Experimenting with distant supervision for emotion classification. 2012 Presented at: 13 th Conference of the European Chapter of the Association for Computational Linguistics; April 23-27, 2012; Avignon, France p. $482-491$.

11. Suttles J, Ide N. Distant supervision for emotion classification with discrete binary values. 2013 Presented at: 14th International Conference on Intelligent Text Processing and Computational Linguistics; March 24-30, 2013; Samos, Greece p. 121-136. [doi: 10.1007/978-3-642-37256-8 11]

12. Sahni T, Chandak C, Chedeti NR, Singh M. Efficient Twitter sentiment classification using subjective distant supervision. 2017 Presented at: 2017 9th International Conference on Communication Systems and Networks (COMSNETS); January 4-8, 2017; Bengaluru, India. [doi: 10.1109/comsnets.2017.7945451]

13. Camacho-Collados J, Doval Y, Martínez-Cámara E, Espinosa-Anke L, Barbieri F, Schockaert S. Learning cross-lingual word embeddings from Twitter via distant supervision. arXiv. Preprint posted online on March 31, 2020 [FREE Full text]

14. Mohammed S, Ghelani N, Lin J. Distant supervision for topic classification of Tweets in curated streams. arXiv. Preprint posted online on April 22, 2017 [FREE Full text]

15. Kim Y. Convolutional neural networks for sentence classification. arXiv. Preprint posted online on September 3,2014 [FREE Full text] 
16. Kalchbrenner N, Grefenstette E, Blunsom P. A convolutional neural network for modelling sentences. arXiv. Preprint posted online on April 8, 2014 [FREE Full text]

17. Dieng AB, Wang C, Gao J, Paisley J. TopicRNN: A recurrent neural network with long-range semantic dependency. arXiv. Preprint posted online on November 5, 2016 [FREE Full text]

18. Devlin J, Chang MW, Lee K, Toutanova K. BERT: Pre-training of deep bidirectional transformers for language understanding. arXiv. Preprint posted online on October 11, 2018 [FREE Full text]

19. Vaswani A, Shazeer N, Parmar N, Uszkoreit J, Jones L, Gomez AN, et al. Attention is all you need. In: Advances in neural information processing systems. 2017 Presented at: 31st International Conference on Neural Information Processing Systems; December 4, 2017; Long Beach, California, USA p. 6000-6010.

20. Bengio Y, Ducharme R, Vincent P, Jauvin C. A neural probabilistic language model. J Mach Learn Res 2003 Feb;3:1137-1155 [FREE Full text] [doi: 10.5555/944919.944966]

21. Mikolov T, Chen K, Corrado G, Dean J. Efficient estimation of word representations in vector space. arXiv. Preprint posted online on September 7, 2013 [FREE Full text]

22. Mikolov T, Sutskever I, Chen K, Corrado G, Dean J. Distributed representations of words and phrases and their compositionality. arXiv. Preprint posted online on October 16, 2013 [FREE Full text]

23. Pennington J, Socher R, Manning C. Glove: Global vectors for word representation. 2014 Presented at: 2014 Conference on Empirical Methods in Natural Language Processing (EMNLP); October 2014; Doha, Qatar p. 1532-1543. [doi: $10.3115 / \mathrm{v} 1 / \mathrm{d} 14-1162]$

24. Huang Z, Hu Q, Gu J, Yang J, Feng Y, Wang G. Web-based intelligent agents for suicide monitoring and early warning. China Digital Medicine 2019;14(3):2-6.

25. Yang F, Huang Z, Yang B, Ruan J, Nie W, Fang S. Analysis of suicidal ideation patterns weibo "tree hole" users based on artificial intelligence technology. Journal of Nursing Science 2019;34(24):42-45 [FREE Full text]

26. Glorot X, Bordes A, Bengio Y. Deep sparse rectifier neural networks. 2011 Presented at: 14th International Conference on Artificial Intelligence and Statistics (AISTATS); April 11-13, 2011; Ft. Lauderdale, FL, USA URL: http://proceedings. mlr.press/v15/glorot11a/glorot11a.pdf

27. Ioffe S, Szegedy C. Batch normalization: Accelerating deep network training by reducing internal covariate shift. arXiv. Preprint posted online on February 11, 2015 [FREE Full text]

28. Guan L, Hao B, Cheng QJ, Ye Z, Zhu T. Behavioral and linguistic characteristics of microblog users with various suicide ideation level: An explanatory study. Chinese Journal of Public Health 2015;31(3):349-352.

29. Ryan T, Xenos S. Who uses Facebook? An investigation into the relationship between the Big Five, shyness, narcissism, loneliness, and Facebook usage. Comput Human Behav 2011 Sep;27(5):1658-1664. [doi: 10.1016/j.chb.2011.02.004]

30. Gosling SD, Augustine AA, Vazire S, Holtzman N, Gaddis S. Manifestations of personality in online social networks: self-reported Facebook-related behaviors and observable profile information. Cyberpsychol Behav Soc Netw 2011 Sep;14(9):483-488 [FREE Full text] [doi: 10.1089/cyber.2010.0087] [Medline: 21254929]

31. Cash SJ, Thelwall M, Peck SN, Ferrell JZ, Bridge JA. Adolescent suicide statements on MySpace. Cyberpsychol Behav Soc Netw 2013 Mar;16(3):166-174. [doi: 10.1089/cyber.2012.0098] [Medline: 23374167]

32. Spates K, Ye X, Johnson A. "I just might kill myself": Suicide expressions on Twitter. Death Stud 2020;44(3):189-194. [doi: 10.1080/07481187.2018.1531085] [Medline: $\underline{\text { 30556789] }}$

33. Zoufan. Weibo. URL: https://www.weibo.com/xiaofan116?is all=1 [accessed 2021-07-06]

34. Weibo. Weibo. URL: https://www.weibo.com/ [accessed 2021-07-06]

35. Kingma DP, Ba J. Adam: A method for stochastic optimization. arXiv. Preprint posted online on December 22, 2014 [FREE Full text]

36. Paszke A, Gross S, Massa F, Lerer A, Bradbury J, Chanan G, et al. PyTorch: An imperative style, high-performance deep learning library. 2019 Presented at: Advances in Neural Information Processing Systems 32 (NeurIPS 2019); December 8-14, 2019; Vancouver, BC, Canada.
Abbreviations
BERT: bidirectional encoder representations from transformers
DCG: definite clause grammar 
Edited by R Kukafka; submitted 28.11.20; peer-reviewed by X Xiu, A Teles; comments to author 26.01.21; revised version received 24.03.21; accepted 04.05.21; published 26.08.21

Please cite as:

$F u$ G, Song C, Li J, Ma Y, Chen P, Wang R, Yang BX, Huang Z

Distant Supervision for Mental Health Management in Social Media: Suicide Risk Classification System Development Study

J Med Internet Res 2021;23(8):e26119

URL: $\underline{\text { https://www.jmir.org/2021/8/e26119 }}$

doi: $10.2196 / 26119$

PMID: $\underline{34435964}$

(C) Guanghui Fu, Changwei Song, Jianqiang Li, Yue Ma, Pan Chen, Ruiqian Wang, Bing Xiang Yang, Zhisheng Huang. Originally published in the Journal of Medical Internet Research (https://www.jmir.org), 26.08.2021. This is an open-access article distributed under the terms of the Creative Commons Attribution License (https://creativecommons.org/licenses/by/4.0/), which permits unrestricted use, distribution, and reproduction in any medium, provided the original work, first published in the Journal of Medical Internet Research, is properly cited. The complete bibliographic information, a link to the original publication on https://www.jmir.org/, as well as this copyright and license information must be included. 